\title{
HIV and SIV Induce Alterations in CNS CaMKII Expression and Activation
}

\section{A Potential Mechanism for Cognitive Impairment}

Ravi G. Gupta, ${ }^{*}$ Kathleen M. Kelly, ${ }^{\star \dagger}$ Kris L. Helke, ${ }^{*}$ Suzanne E. Queen, ${ }^{*}$ Jami M. Karper, ${ }^{*}$ Jamie L. Dorsey, ${ }^{*}$ Angela K. Brice, ${ }^{*}$ Robert J. Adams, ${ }^{*}$ Patrick M. Tarwater, ${ }^{\ddagger}$ Dennis L. Kolson, $§$ and Joseph L. Mankowski ${ }^{*+\pi}$

From the Departments of Molecular and Comparative Pathobiology, ${ }^{*}$ Pathology, ${ }^{\dagger}$ and Neurology, ${ }^{\top 1}$ Johns Hopkins University School of Medicine, Baltimore, Maryland; the Division of Biostatistics and Epidemiology, Paul L. Foster School of Medicine, El Paso, Texas; and the Departments of Neurology and Microbiology, ${ }^{\$}$ University of Pennsylvania, Philadelphia, Pennsylvania

The molecular mechanisms underlying learning and memory impairment in patients with HIV-associated neurological disease have remained unclear. Calcium/calmodulin-dependent kinase II (CaMKII) has key roles in synaptic potentiation and memory storage in neurons and also may have immunomodulatory functions. To determine whether HIV and simian immunodeficiency virus (SIV) induce alterations in CaMKII expression and/or activation (autophosphorylation) in the brain, we measured CaMKII alterations by quantitative immunoblotting in both an in vitro HIV/ neuronal culture model and in vivo in an SIV-infected macaque model of HIV-associated neurological damage. Using primary rat hippocampal neuronal cultures treated with culture supernatants harvested from HIV-1-infected human monocyte-derived macrophages (HIV/MDM), we found that CaMKII activation declined after exposure of neurons to HIV/MDM. Consistent with our in vitro measurements, a significant decrease in CaMKII activation was present in both the hippocampus and frontal cortex of SIV-infected macaques compared with uninfected animals. In SIV-infected animals, total CaMKII expression in the hippocampus correlated well with levels of synaptophysin. Furthermore, CaMKII expression in both the hippocampus and frontal cortex was inversely correlated with viral load in the brain. These findings suggest that alterations in CaMKII may compromise synaptic function in the early phases of chronic neurodegenerative processes induced by HIV. (Am J Pathol 2010, 176:2776-2784; DOI: 10.2353/ajpath.2010.090809)

More than 30 million people worldwide are infected with the human immunodeficiency virus (HIV; World Health Organization UNAIDS 2008). Although highly active antiretroviral therapies have dramatically improved survival rates and health-related quality of life for patients, HIVassociated neurocognitive disorders remain a significant public health problem. ${ }^{1}$ In the highly active antiretroviral therapies era, the prevalence of less severe forms of HIVassociated neurocognitive disorders has increased in individuals who are not immunosuppressed. ${ }^{2,3}$ Learning and memory impairment is common in HIV-infected individuals with estimates ranging from 40 to $60 \%$ of patients. ${ }^{4,5}$ Synaptodendritic injury has emerged as a possible cause of these deficits in HIV, as well as other neurodegenerative diseases including Alzheimer disease, Parkinson disease, and schizophrenia. ${ }^{6-9}$

In particular, studies have implicated altered neuronal synaptic transmission and plasticity changes in the hippocampus as the mechanism underlying the cognitive impairment observed in HIV patients. In a murine model of HIV encephalitis (HIVE), for example, neuronal damage in the hippocampus was associated with impaired spatial learning. ${ }^{10}$ Neuropsychological tests in rhesus monkeys infected with simian immunodeficiency virus

Supported by National Institutes of Health grant HL078479 (to J.L.M.) and $\mathrm{NIH}$ grant RR007002 (to K.M.K., K.L.H., and A.K.B.). R.G.G. was partially supported by the Johns Hopkins University Provost's Undergraduate Research Award Program.

Accepted for publication January 28, 2010

Current address for K.L.H.: Department of Comparative Medicine, Medical University of South Carolina, Charleston, SC.

Address reprint requests to Joseph L. Mankowski, D.V.M., Ph.D., Department of Molecular and Comparative Pathobiology, Johns Hopkins University, 733 N. Broadway, BRB 827, Baltimore, MD 212052196. E-mail: jmankows@jhmi.edu. 
(SIV) have demonstrated similar functional impairment attributable to the hippocampus, consistent with psychomotor and memory impairment observed in HIV-infected humans. ${ }^{11}$ However, the mechanisms underlying HIV-induced alterations in synaptic plasticity and longterm potentiation (LTP), the synaptic mechanism of memory storage, in the hippocampus have not been defined.

Calcium/calmodulin-dependent kinase II (CaMKII) is necessary for the induction of LTP in the hippocampus. Once activated by calcium flux through the $\mathrm{N}$-methyl$D$-aspartic acid (NMDA) channel, CaMKII initiates the biochemical cascade that potentiates synaptic transmission. The kinase functions as a molecular switch with stable "on" (phosphorylated) and "off" (unphosphorylated) states, a mechanism that may underlie synaptic potentiation and memory storage in neurons. ${ }^{12}$ In addition to serving as a transducer in LTP induction, CaMKII is also responsible for the persistence of LTP, a crucial aspect of memory. ${ }^{13}$ Autophosphorylation of threonine 286 of CaMKII is critical for persistent activation of CaMKII as mutation of this site eliminates phosphorylation and also blocks LTP. ${ }^{14}$ This mutation also interferes with experience-dependent plasticity in vivo and with memory, demonstrating an important consolidating role for CaMKII in cognitive function. ${ }^{15-17}$

Given the diverse roles that CaMKII plays in neuronal development, maturation, and plasticity, changes in CaMKII expression and activity may have significant implications for the pathophysiology of HIV CNS disease. In particular, HIV-induced alterations in neuronal CaMKII expression and activation may impair synaptodendritic function in key brain regions. To determine whether HIV induces alterations in neuronal CaMKII expression and activation in the hippocampus, we examined CNS CaMKII alterations both in vitro and in vivo. Cultured rat hippocampal neurons were treated with supernatants harvested from HIV-infected human monocyte-derived macrophages, an established in vitro model of HIV-induced neuronal damage, to measure HIV-induced alterations in CaMKII protein in neurons. ${ }^{18,19}$ We also studied CaMKII alterations in the CNS using an SIV/macaque model of HIV-associated neurological damage. CaMKII expression and activation were measured in the hippocampus and frontal cortex of 12 rhesus macaques inoculated with the neurovirulent clone SIV/17E-Fr and the immunosuppressive strain SIV/DeltaB670. Synaptophysin expression, an established marker of synaptic integrity, was measured in SIV-infected macaques to determine whether CaMKII alterations are a molecular correlate of synaptic pathology in HIV CNS disease. CNS viral replication and host inflammatory responses were also evaluated to identify factors that potentially mediate alterations in CNS CaMKII expression and activation.

\section{Materials and Methods}

\section{Animals}

This study evaluated 15 rhesus macaques (Macaca mulatta), including 12 that were intravenously inoculated with both SIV/DeltaB670 (50 $\left.\mathrm{AID}_{50}\right)$ and SIV/17E-Fr $\left(10,000 \mathrm{AID}_{50}\right)$ as previously described. ${ }^{20-22}$ Three additional uninfected mock-inoculated (media alone) macaques served as controls. The average ages of SIVinfected and uninfected macaques were 11.2 years and 11.3 years, respectively. Animal survival (i.e., progression to AIDS) in the SIV-infected group varied-animals that did not develop encephalitis survived an average of 334 days postinoculation, whereas macaques that did develop encephalitis survived an average of 100 days postinoculation. At time of euthanasia, animals were perfused with sterile saline to remove blood and blood-borne leukocytes from the CNS vasculature. Brains were immediately harvested, and samples were either immersion fixed in STF (Streck, Omaha, NE) or rapidly frozen for isolation of RNA and preparation of protein homogenates. The animal procedures in this study were reviewed and approved by the Johns Hopkins University Institutional Animal Care and Use Committee in accordance with Animal Welfare Act regulations and the USPHS Policy on Humane Care and Use of Laboratory Animals.

Hematoxylin and eosin-stained sections of frontal and parietal cortex, basal ganglia, thalamus, midbrain, and cerebellum from each animal were examined microscopically in a blinded fashion by two pathologists (K.K. and K.H.). SIV-infected macaques with encephalitis developed typical HIV/SIV CNS lesions consisting of multifocal perivascular accumulations of SIV-laden macrophages and multinucleated giant cells in white matter, basal ganglia, and thalamus. Animals with classic histopathological lesions of HIV/SIV encephalitis (SIVE, $n=6$ animals) were designated as SIVE + . Animals that did not develop characteristic lesions ( $n=6$ animals) were categorized as SIVE-. CNS lesions were not present in the uninfected macaques ( $n=3$ animals).

\section{Immunohistochemical Staining}

To identify cellular localization of CaMKII in macaque brain, coronal sections of hippocampus were immunohistochemically stained with a monoclonal antibody directed against CaMKII protein (clone 6G9, 1:1000, Millipore, Billerica, MA). Primary antibody against CD68 was used as a marker of macrophage infiltration and microglial activation in coronal sections including basal ganglia and frontal cortex (1:2000, clone KP-1, DAKO, Carpinteria, CA). For uniformity, all brain tissue sections were stained using an automated immunostainer (Optimax Plus, BioGenex, San Ramon, CA). Paraffin-embedded brain tissue sections were deparaffinized and rehydrated. After rinsing in water, tissues were heated in a microwave in sodium citrate buffer $(0.01 \mathrm{~mol} / \mathrm{L}, \mathrm{pH}$ 6.0) for 8 minutes to retrieve antigen. Endogenous peroxidase was quenched with $3 \% \mathrm{H}_{2} \mathrm{O}_{2}$ for 10 minutes, and then sections were blocked with buffered casein for 10 minutes. Primary antibody was applied to tissue sections for 60 minutes at room temperature, the tissues were washed in buffer, and then secondary biotinylated multilink antibody (BioGenex) was added for 20 minutes. After washing, streptavidin-horseradish peroxidase was applied for 20 
minutes, followed by diaminobenzidine tetrahydrochloride in buffer containing $\mathrm{H}_{2} \mathrm{O}_{2}$ for 10 minutes. Sections were then washed, dehydrated, and mounted. Control experiments included omission of primary antibodies using the same staining protocol and were devoid of specific immunoreaction product.

\section{Viral Load in Brain}

To measure SIV RNA levels in the CNS, real-time RT-PCR was performed on RNA isolated from basal ganglia. At postmortem, samples were snap frozen in liquid nitrogen for RNA isolation as previously described. ${ }^{20}$ RNA was isolated from $50 \mathrm{mg}$ of brain tissue using the RNA STAT-60 Kit (Tel-Test "B", Inc.) and an RNeasy MiniKit (Qiagen, Valencia, CA). Quantitation of SIV RNA in extracted brain tissue was done with SIV gag region primers and probe. Real-time RT-PCR detected both cellassociated full-length viral transcripts and genomic RNA present in tissue-associated virions.

\section{HIV/Neuronal Cell Cultures}

Human monocyte-derived macrophages (MDM) were isolated from healthy volunteers as previously described. ${ }^{23}$ Cells were collected in accordance with protocols approved by the University of Pennsylvania Committee on Studies Involving Human Beings. Macrophages were grown in six-well plates $\left(1.25 \times 10^{6}\right.$ cells per well) for 7 days and then inoculated with equivalent amounts of HIV-1 (100 ng of p24 per well). Infected cells were incubated for 18 hours. The HIV-1 strain used, Jago, is a macrophage-tropic isolate derived from the cerebrospinal fluid from a patient with confirmed HIV-associated dementia. ${ }^{18,23}$ Productive infection of macrophages was confirmed by serial quantification of p24 antigen levels in the culture supernatants over time. Supernatants were used for application to neuronal cultures only when productive infection was confirmed (p24 values between 100 to $400 \mathrm{pg} / \mathrm{ml}$ supernatant).

Primary rat hippocampal cultures were prepared from embryonic day 15 Sprague-Dawley rat pups as previously described. ${ }^{23}$ Cells were plated at a density of $2 \times$ $10^{5}$ cells per $35-\mathrm{mm}$ dish on glass coverslips. Neuronal cultures were treated with HIV/MDM supernatants at a final dilution of $1: 10$ at $37^{\circ} \mathrm{C}, 5 \% \mathrm{CO}_{2}$. Cultures were washed twice with cold PBS and lysed in ice-cold Laemmli buffer (0.625 M Tris- $\mathrm{HCl}$, pH 6.8, 2\% SDS, and 10\% glycerol) at selected time points after exposure to supernatants ( 5 minutes, 15 minutes, 30 minutes, 1 hour, and 4 hours) for CaMKII analysis. Neuronal cultures treated with mock/MDM supernatants were used for control comparison. An untreated neuronal culture lysate served as a gel-to-gel standard.

\section{Quantitative Western Blotting}

To perform quantitative immunoblotting, hippocampus and frontal cortex tissue samples from uninfected and SIV-infected macaques were homogenized in $50 \mathrm{mmol} / \mathrm{L}$
Tris, $150 \mathrm{mmol} / \mathrm{L} \mathrm{NaCl}, 0.1 \%$ SDS, $1.0 \%$ NP-40, $0.5 \%$ $\mathrm{Na}$-desoxycholate, and $\mathrm{dH}_{2} \mathrm{O}$. Homogenates were triturated 5 times through a 20-gauge needle and spun at $14,000 \mathrm{rpm}$ for 20 minutes at $4^{\circ} \mathrm{C}$. Protein concentration in supernatants was quantitated at 1:1000 dilution with a Bio-Rad Protein Assay kit, which is based on the Bradford method and standard curve analysis.

Tissue homogenates were boiled for 3 minutes, and equivalent amounts $(30 \mu \mathrm{g})$ of sample were loaded onto 10\% Bis-Tris polyacrylamide gels and SDS-PAGE was performed. Cell lysates from in vitro cultures were prepared using the same protocol. Gels were blotted onto Immunoblot polyvinylidene difluoride membranes (Bio-Rad, Hercules, CA) and blocked in PBS and 5\% nonfat milk in PBS for 1 hour at room temperature. Primary antibodies included (1) pan-CaMKII polyclonal antibody (1:1000, catalog \#3362), (2) anti-phosphorylated (Thr286) CaMKII polyclonal antibody (1:1000, catalog \#3361), and (3) $\beta$-actin polyclonal antibody (1:10000, catalog \#4967; Cell Signaling Technology, Beverly, MA). The P-CaMKII antibody detects endogenous levels of CaMKII only when phosphorylated at threonine 286, consistent with CaMKII activation. The density of neuronal synapses in the hippocampus was measured using a monoclonal antibody against synaptophysin (1:5000, catalog \# IR776, DAKO) in immunoblots. Primary antibodies were incubated overnight at room temperature in blocking solution. Membranes were washed four times in PBS with $0.1 \%$ Tween-20 (PBS-Tween) and incubated in secondary antibody conjugated to HRP $(125 \mathrm{ng} / \mathrm{ml}$, Cell Signaling Technology) in blocking solution for 1 hour at room temperature. Membranes were washed six times with PBS-Tween, developed in LumiGLO chemiluminescent substrate (Cell Signaling Technology), and exposed to Basic Autorad film. For densitometric analysis, films were scanned into Adobe Photoshop (Adobe Systems, San Jose, CA), and a fixed cursor area centered over each band was assessed for pixel density, with normalization to $\beta$-actin signal. To allow for comparison of measurements between gels, a brain homogenate obtained from a control animal was included on all gels. Serial dilutions of this sample, ranging from 4 to $60 \mu \mathrm{g}$ of protein, were also assessed under the same conditions to ensure that all measurements of CaMKII and actin were within the linear range for measurement as we have described previously. ${ }^{21}$

\section{Statistical Analysis}

All statistical inferences ( $P$ values) were calculated using parametric methods. To determine whether exposure to HIV-infected macrophages induced changes in CaMKII levels in hippocampal neurons, two replicate neuronal lysates were harvested for each treatment group (mockexposed control and HIV-exposed) at five time points posttreatment. To test for significant differences between these two groups, we compared trends over time using a linear regression model to calculate a $P$ value for the differences between the slopes (change over time) of the mock and HIV groups. The slopes of linear regression lines were compared using an interaction term between 
change over time and group membership, a common statistical method. For analysis of CNS CaMKII levels in macaques, mean total CaMKII and activated CaMKII levels in three subsets of animals (uninfected, SIVE-, SIVE+) were compared using one-way analysis of variance. Subsequent comparisons between any two of the three groups used the Bonferroni method to adjust for multiple comparisons. The Pearson correlation coefficient was used to analyze the relationships between CaMKII and synaptophysin protein expression, as well as CaMKII expression and CNS viral load in SIV-infected animals. In all analyses, statistical significance was defined as a $P$ value of less than 0.05

\section{Results}

\section{Treatment of Cultured Hippocampal Neurons with Culture Supernatant from HIV-Infected Macrophages Induces Early and Sustained Loss of CaMKII Activation}

To determine whether HIV-1 infection induces alterations in CaMKII expression and/or activation in the hippocampus, we first evaluated CaMKII levels in an in vitro model of HIV CNS disease. Cultures containing primary rat hippocampal neurons were treated with cell culture supernatants harvested from HIV-1-infected monocyte-derived macrophages (HIV/MDM) as previously described. ${ }^{18,19,23}$ Neurons treated with supernatants collected from mock-infected macrophages were prepared for control comparison. Cells were lysed at successive time points after exposure to supernatants (5, 15, 30, 60, and 240 minutes posttreatment) to obtain two replicate protein samples for measurements. At each time point, levels of both total and activated (phosphorylated, Thr286) CaMKII present were measured by quantitative immunoblotting. CaMKII signal was normalized to in-lane $\beta$-actin to control for potential variability in protein loading for all measurements. To test for significant differences between mock and HIV-treated neuron cultures, we compared CaMKII trends over time using a linear regression model that enabled us to calculate $P$ values for the differences between the slopes (change over time) between mock and HIV supernatant groups. A 50-kDa band corresponding to CaMKII protein was present in cell lysates. The level of total CaMKII in neurons was not significantly altered by the addition of cell culture supernatants from HIV-infected macrophages at any time point posttreatment (Figures 1 and 2A, $P=0.071$ ). As the latest time point at which neurons were lysed was 4 hours after addition of HIV/MDM supernatants, changes in protein expression were not expected.

Impaired calcium homeostasis has been demonstrated in other models of excitotoxic neuronal degeneration. ${ }^{24,25}$ As calcium dysregulation also may develop in HIV neurotoxicity, HIV-induced alterations of calcium signal transduction may occur relatively early in hippocampal neurons after addition of HIV/MDM supernatants and would be reflected by a decrease in CaMKII activation. CaMKII activation, represented by the amount of phosphorylated CaMKII in im-
HIV/MDM supernatant-treated (time post-treatment)

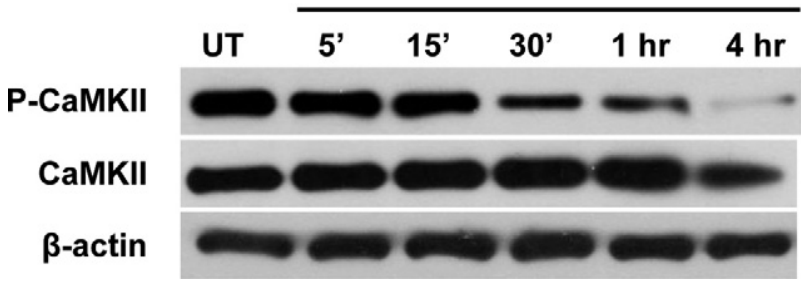

Figure 1. Treatment of primary neuron cultures with supernatant from HIV-infected macrophages alters CaMKII activation. Western blots performed on cell lysates of primary neuron cultures treated with HIV/MDM culture supernatants demonstrated that activation of CaMKII (represented by phosphorylation of CaMKII $=$ P-CaMKII) declined beginning 30 minutes posttreatment and continuing to the 4-hour end point. In contrast, total CaMKII levels did not differ over time. UT indicates untreated neuronal cultures.

munoblots, began to decline 30 minutes after exposure to HIV/MDM supernatants and decreased further from 30 minutes to 4 hours postexposure. (Figure 2B, $P=0.015$ ). In parallel, a significant decrease in the ratio of activated to total CaMKII developed at 30 minutes and then decreased further until the 4-hour end point after supernatant exposure (Figure 2C; $P=0.016$ ). These findings show that mediators in supernatants from HIV-infected human macrophages induced early and sustained inhibition of CaMKII activation in cultured rat hippocampal neurons. In addition, the fraction of the total CaMKII pool that was activated significantly declined over time after exposure to supernatants from HIV-infected human macrophages.

\section{CaMKII Expression in Neurons of SIV-Infected Macaques}

Our in vitro measurements indicated that neuronal CaMKII may be an important mediator of synaptic injury in the hippocampus. Although synaptic damage correlates closely with the presence and severity of cognitive impairment in $\mathrm{HIV},{ }^{26}$ the molecular and cellular correlates of neurocognitive dysfunction have been difficult to characterize in humans. To address this, we measured CNS CaMKII alterations in vivo using the SIV/macaque model.

We first evaluated sections of hippocampus from SIVinfected rhesus macaques by immunostaining for CaMKII and the macrophage marker CD68 to determine whether neurons express high levels of CaMKII in the CNS with the development of SIV encephalitis. Rhesus macaques inoculated with SIV/17E-Fr and SIV/DeltaB670 with SIV encephalitis (SIVE+) had multifocal perivascular accumulations of CD68+ macrophages and multinucleated giant cells in the hippocampus as well as basal ganglia and subcortical white matter consistent with CNS lesions that develop in SIV-infected pigtailed macaques after inoculation with SIV/ 17E-Fr and SIV/DeltaB670 (Figure 3, A and B). ${ }^{22}$ In the hippocampus, CaMKII was highly expressed in neurons (Figure 3, C and D) rather than microglia, infiltrating macrophages, or multinucleated giant cells (Figure 3D). Therefore, measurements of CaMKII performed on brain homogenates from the hippocampus and the frontal cortex are primarily representative of alterations in neuronal CaMKII levels rather than inflammatory or glial cells in the CNS. 
A

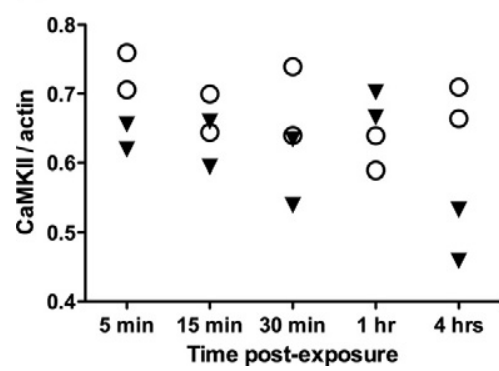

B

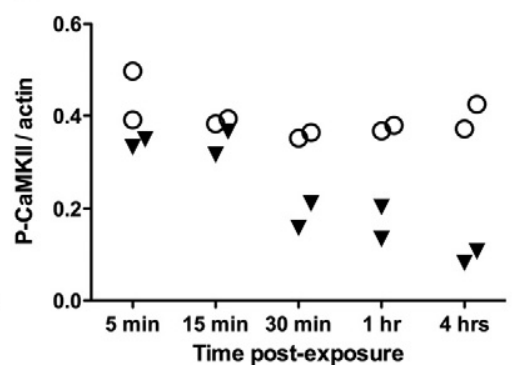

C

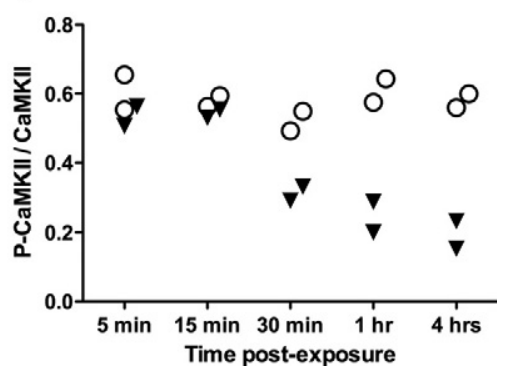

Figure 2. HIV induces early and sustained inhibition of CaMKII activation in hippocampal neurons. To measure CaMKII levels and activation via immunoblotting, primary rat hippocampal neurons were lysed at successive time points after exposure to HIV(Jago)/MDM (triangles). Mock/MDM-exposed neurons (circles) were prepared for control comparison at each time point. A: Total CaMKII was not altered in HIV/MDM-exposed neurons. B: In contrast, CaMKII activation (autophosphorylation) began declining 30 minutes after exposure to HIV/MDM and continued to decrease until 4 hours postexposure $(P=0.015)$. C: The ratio of active to total CaMKII also decreased beginning at 30 minutes postexposure and continuing to the 4-hour postexposure time point $(P=0.016)$

\section{SIV Infection Alters Hippocampal CaMKII in Vivo}

CaMKII expression and activation in the hippocampus of SIV-infected macaques (uninfected, SIVE-, SIVE+) were measured by SDS-PAGE and quantitative immunoblotting. Total CaMKII levels were increased in SIV-infected macaques compared with uninfected controls, with highest levels present in SIV-infected animals without encephalitis (Figure 4A; $P=0.001$ ). Although total CaMKII levels were lower in SIV-infected animals with encephalitis $(P=0.011)$ compared with SIV-infected macaques that did not develop encephalitis, the SIVE+ levels still remained significantly higher than uninfected control animals $(P=0.017)$.

Consistent with our in vitro observations in neuronal cultures, hippocampal CaMKII activation significantly decreased in SIV-infected animals compared with controls (Figure 4B). CaMKII activation was significantly reduced in the SIVE- group $(P=0.048)$ versus controls. With the development of SIVE, the decrease in CaMKII activation was greater (Figure 4B; $P=0.001$ versus uninfected controls, $P=0.042$ versus SIVE- group).
To determine how alterations in CaMKII activation and expression were related, we compared the ratio of activated CaMKII to the total CaMKII levels in the hippocampus (Figure 4C). Although total CaMKII increased in SIVinfected animals, the ratio of activated to total CaMKII declined in SIV-infected macaques compared with controls $(P<0.001)$. These data indicate that SIV CNS infection alters both CaMKII expression and activation patterns in the hippocampus.

\section{SIV-Induced CaMKII Alterations in the Frontal Cortex Parallel Hippocampal Changes}

In addition to serving crucial roles in hippocampal neuronal function, CaMKII also modulates synaptic events required for the consolidation of memory traces in cortical networks. ${ }^{17}$ Although cortical synaptodendritic atrophy is common in HIV, the specific causes of these progressive degenerative effects remain unknown. CaMKII alterations may be the molecular basis for these deficits. To deter-

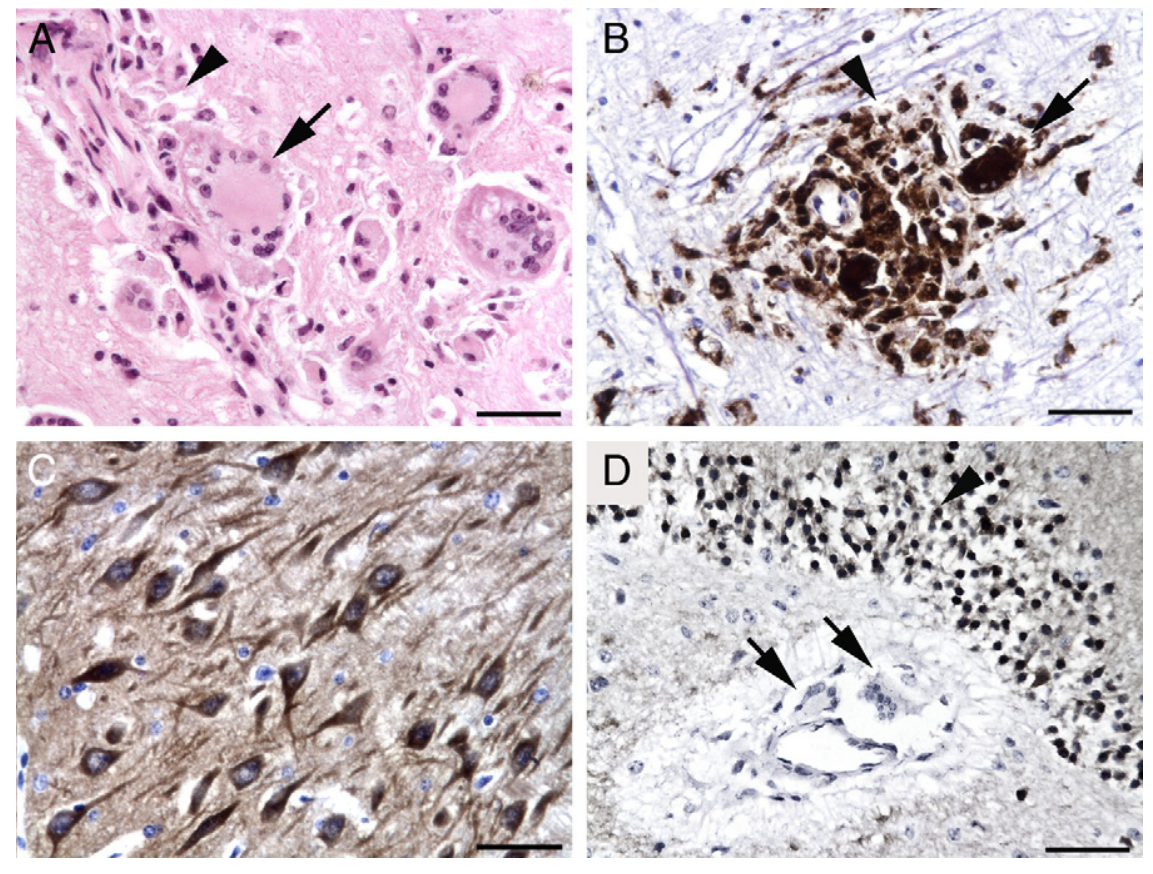

Figure 3. In SIV-infected rhesus macaques with encephalitis, CaMKII is highly expressed in hippocampal neurons. Rhesus macaques with SIV encephalitis had multifocal perivascular accumulations of macrophages (arrowhead) and multinucleated giant cells (arrow; A, hematoxylin and eosin stain). These macrophages (arrowhead) and multinucleated giant cells (arrow) were immunopositive for the macrophage marker $\mathrm{CD} 68$ (brown, B). CaMKII immunoreactivity (brown) in the hippocampus of macaques with encephalitis was localized to neurons $(\mathbf{C})$. In contrast, macrophage and multinucleated giant cell CaMKII immunostaining (arrows) was minimal (D). Scale bars $=50 \mu \mathrm{m}$. 
A

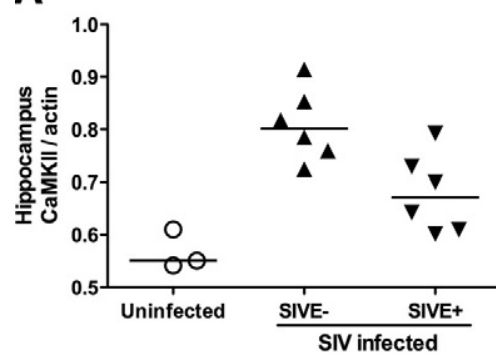

D

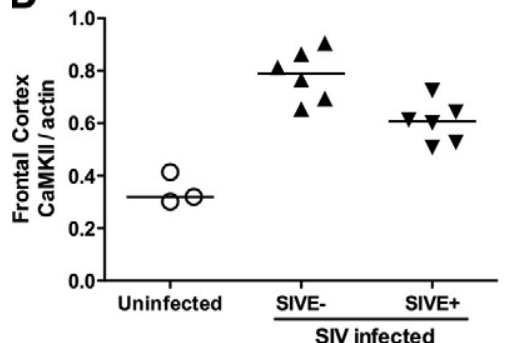

B

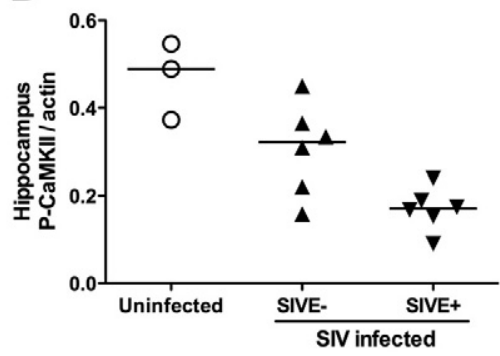

C

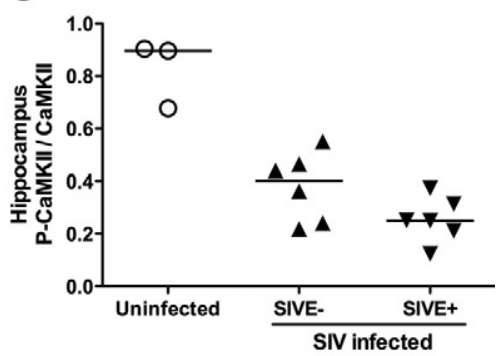

$\mathbf{F}$

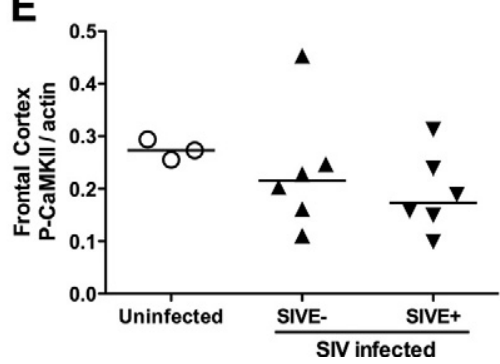

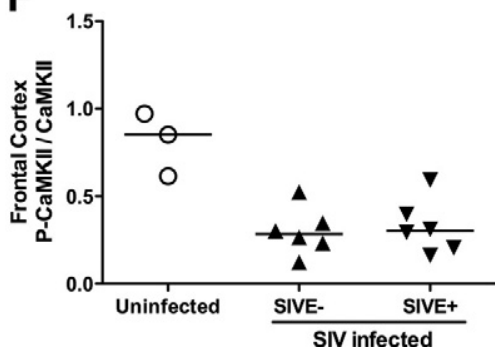

Figure 4. CaMKII expression and activation is altered in both hippocampus and frontal cortex of SIV-infected macaques. Total CaMKII was increased in the hippocampus of SIV-infected rhesus macaques (A). Although expression decreased in animals that developed encephalitis (SIVE+), total CaMKII remained significantly higher in SIV-infected animals compared with uninfected controls. In contrast, CaMKII activation in hippocampus was inhibited by SIV infection (B). Overall, in hippocampus, the ratio of active to total CaMKII declined in SIV-infected animals despite the increase in total CaMKII (C). Similar changes in CaMKII expression and activation were identified in the frontal cortex of SIV-infected macaques. CaMKII expression was increased in SIV-infected animals but then declined with the development of SIV encephalitis (D), whereas CaMKII activation decreased in SIV-infected animals with no significant change detected in the SIVE + subset (E). The P-CaMKII/CaMKII ratio in frontal cortex also deceased in SIV-infected macaques (F), indicating that SIV infection inhibits CaMKII activity in both the hippocampus and frontal cortex of SIV-infected macaques.

mine whether SIV induced changes in cortical CaMKII levels, we measured CaMKII expression and activation in the frontal cortex of SIV-infected macaques by SDSPAGE and quantitative immunoblotting.

Similar to our observations in the hippocampus, total CaMKII was increased in the frontal cortex of SIV-infected macaques compared with controls (Figure 4D), with highest levels present in SIV-infected animals without encephalitis $(P<0.001)$. Total CaMKII levels were lower in SIV-infected animals with encephalitis $(P=0.009)$ compared with SIVinfected macaques that did not develop encephalitis, but the SIVE+ CaMKII levels remained significantly higher than uninfected control animals $(P=0.003)$.

Although CaMKII activation was lower in SIV-infected animals (Figure 4E), the decline in CaMKII activation was not significant. However, the ratio of activated to total CaMKII was significantly decreased in SIV-infected macaques compared with uninfected controls (Figure 4F; SIVE- versus controls, $P=0.001$; SIVE + versus controls, $P=0.002)$. These data demonstrate that alterations in CaMKII expression and activation extend beyond the hippocampus to the frontal cortex of SIV-infected macaques.

\section{CaMKII Alterations Are Associated with Synaptophysin Loss in the Hippocampus}

Measurement of synaptophysin, a calcium-binding synaptic protein involved in neurotransmitter release, is useful for evaluating synaptic density as it reflects synaptic integrity. ${ }^{27-30} \mathrm{~A}$ decrease in synaptophysin suggests synaptic damage and has been associated with cognitive decline in several chronic neurodegenerative diseases. ${ }^{31-34}$ We measured synaptophysin expression by quantitative immunoblotting to evaluate synaptic integrity in the hippocampus of SIVinfected macaques. To examine the relationship between CaMKII and synaptophysin protein expression, we compared total CaMKII with synaptophysin levels in the hippocampus. CaMKII expression was strongly correlated with synaptophysin expression (Figure $5 ; r=0.62, P=0.042$, Pearson correlation coefficient). These data demonstrate that SIV-induced alterations in CaMKII expression are associated with decreased synaptic density in the hippocampus.

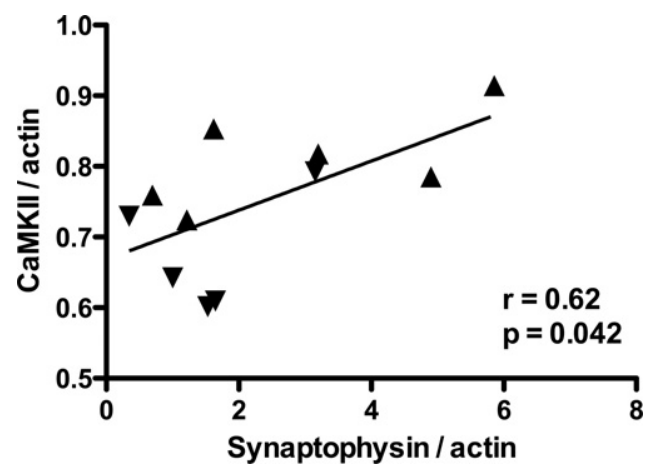

Figure 5. Altered CaMKII expression was correlated with synaptophysin loss in the hippocampus. We measured synaptophysin expression by quantitative immunoblotting to evaluate synaptic density in the hippocampus of SIV-infected macaques. CaMKII expression was strongly correlated with hippocampal synaptophysin levels $(r=0.64, P=0.046$, Pearson correlation coefficient). SIVE - animals represented by triangle symbols pointing up; SIVE + animals represented by triangles pointing down 
A

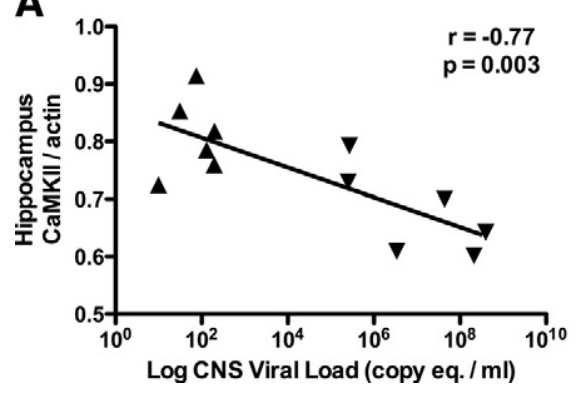

B

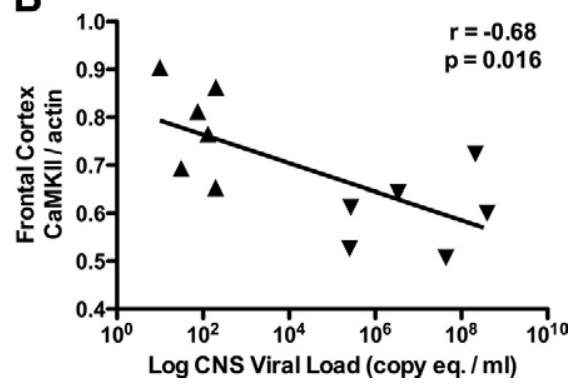

Figure 6. CaMKII protein expression levels were correlated with viral load in the brain. In both the hippocampus (A) and frontal cortex (B) of SIV-infected macaques, the amount of CaMKII expression was significantly inversely correlated with CNS viral load $(\mathrm{r}=-0.77, P=0.033$ and $\mathrm{r}=-0.68, P=0.016$ respectively; Pearson correlation coefficient). SIVE - animals represented by triangle symbols pointing up; SIVE + animals represented by triangles pointing down.

\section{CaMKII Expression Is Inversely Correlated with CNS SIV Replication}

Our measurements of total CaMKII in the hippocampus and frontal cortex suggested that CaMKII expression in SIV-infected animals decreased with progression of SIV CNS disease. To examine the relationship between CaMKII expression and CNS viral replication, we compared levels of total CaMKII in the hippocampus and frontal cortex with levels of SIV RNA measured by real-time RT-PCR. Total CaMKII levels in both the hippocampus and the frontal cortex were strongly inversely correlated with CNS SIV RNA levels (Figure 6, $A$ and $B, P=0.033, r=-0.77$, in the hippocampus; $P=0.016, r=-0.68$, in the frontal cortex; Pearson correlation coefficient). These data indicate that CaMKII expression in the hippocampus and frontal cortex decreases with increasing CNS viral replication. Viral proteins such as gp120 or Tat may regulate CaMKII expression in neurons in conjunction with neurotoxic pro-inflammatory mediators.

\section{Discussion}

These studies demonstrated that SIV infection induced alterations in both CaMKII expression and activation in both the hippocampus and the frontal cortex in the SIV/macaque model of HIV-associated neurocognitive disorders. Using an in vitro $\mathrm{HIV}$ /neuronal culture model, we observed that decreased CaMKII activation was an early and sustained event, beginning within 30 minutes after hippocampal neurons were treated with HIV/MDM supernatants. Although significant neuronal loss was not observed in this model during the time period examined ( 0 to 4 hours postexposure), injury to neuronal synapses may still be substantial. Previous studies using this model suggested that brain regions enriched in NMDA receptors containing the NR2B subunit, such as the hippocampus, are most susceptible to HIV-induced neuronal damage and that calcium-activated neutral protease (calpain)-mediated cleavage of NR2B occurs in HIV infection. ${ }^{18,35}$ Other studies have demonstrated in vitro that overactivation of the NMDA receptor inhibits CaMKII activity in neurons. ${ }^{36}$ As the NR2B subunit is an important synaptic binding partner and regulator of $\mathrm{CaMKII},{ }^{37}$ alterations in CaMKII activation might thus arise from excitotoxic glutamatergic stimulation.

These in vitro observations paralleled measurements made in vivo using the SIV/macaque model, which dem- onstrated that CaMKII activation decreases in SIV-infected animals. Loss of CaMKII in the hippocampus corresponded with decreased synaptophysin expression in SIV-infected animals. Combined with our in vitro observations, these data suggest that CaMKII-associated synaptic injury caused by HIV develop early in infection and is sustained.

We also show that CaMKII expression increased in the hippocampus of SIV-infected animals compared with uninfected controls. CaMKII levels were highest in SIVinfected macaques without encephalitis, which may represent a compensatory response to falling levels of activated CaMKII. With onset of encephalitis, total CaMKII levels are lower, suggesting an inability to maintain CaMKII levels in the face of abundant CNS viral replication and substantial inflammation. The fraction of the total CaMKII pool that was activated in the hippocampus decreased with SIV infection, with the greatest decline occurring in the animals with SIV encephalitis. Overall, these findings demonstrate that the ability to sustain CaMKII activation is compromised with SIV infection despite increases in total CaMKII levels. If SIV encephalitis develops, then maintenance of CaMKII activation becomes even more difficult. Together, these findings indicate that CaMKII alterations may be a molecular correlate of synaptic pathology in SIV CNS disease.

Although we demonstrated a significant correlation between SIV RNA levels in the brain and total CaMKII, other factors, including proinflammatory mediators produced by activated macrophages in the brain, also may contribute to synaptic dysfunction. In HIV and SIV, the covariate nature of macrophage activation and viral replication make it very difficult to separate the effects of virus and inflammatory mediators on neuronal function, especially in studies based on relatively small groups of animals.

In addition to the hippocampus, we also evaluated CaMKII alterations in the frontal cortex of SIV-infected macaques. CaMKII is involved in cortical plasticity and has been shown to have important functions in the consolidation of memories in cortical networks. ${ }^{38,39}$ Total CaMKII was increased in the cortex, whereas the ratio of activated to total CaMKII was significantly decreased. As CaMKII protein is expressed more abundantly in the hippocampus than in the cortex, ${ }^{17}$ loss of normal levels and activation of this kinase may affect cortical plasticity more severely. Our results suggest that alterations in cortical CaMKII activity may be the molecular precursor of syn- 
aptodendritic atrophy in the frontal cortex of HIV-infected individuals.

CaMKII requires cycling between stable "up" (phosphorylated, active) and "down" (unphosphorylated, inactive) states to fulfill its role in memory and long-term neuronal changes. ${ }^{12}$ Our results indicate that SIV infection in the brain disrupts the stability of this molecular switch, perhaps through direct interaction of viral factors with CaMKII. Indeed, previous studies have shown that the Nef protein of HIV-1 binds directly to calcium-bound calmodulin but the physiological significance of this interaction has remained unclear. ${ }^{40}$ These measurements demonstrate a potential functional consequence of viral replication in the hippocampus and imply that a CaMKIImediated pathway may mediate the effects of HIV on cognition.

These results are consistent with previous studies that have highlighted an emerging role for CaMKII in neurodegenerative diseases. Studies have demonstrated in vivo that a loss of CaMKII activity is correlated with neuronal damage during ischemia. ${ }^{41,42}$ Other experiments have used gene knockout mice lacking CaMKII to provide evidence that disruption of this single protein kinase results in damage to neuron dendrites following ischemia. ${ }^{43}$ Moreover, restoring CaMKII activity in hippocampal neurons by treatment with a chemical found in ginseng roots, ginsenoside Rb1, protects and repairs neurons that have been damaged by hypoxia. ${ }^{44}$ In patients suffering from schizophrenia and depression, CaMKII gene expression has also been found to be significantly altered in the hippocampus and frontal cortex. ${ }^{45}$

Loss of synaptic function appears to underlie neurological deficits observed in patients with chronic neurocognitive disorders like HIV-associated neurological disease and Alzheimer disease. ${ }^{46}$ Because synaptic injury may occur long before neuron death and given that surviving neurons have the capacity for synaptic repair leading to functional recovery, therapeutic interventions designed to protect neuron synapses may have great potential for preserving and/or improving neurological function in HIV patients. Although previous studies have shown that disruption of synaptic structure and function occurs after glutamate excitotoxicity and neuroinflammation, ${ }^{7,47}$ the molecular mechanisms underlying synaptic injury in HIV-associated neurocognitive disorders and similar disorders have remained unclear. We have shown that CaMKII may be a novel mediator of synaptic injury in HIV/SIV infection in the brain. Neuronal CaMKII could be an ideal therapeutic target for synaptic repair to improve neurological function in HIV-associated cognitive disease. It will be important to evaluate CaMKII expression and activation in neurodegenerative diseases in addition to HIV CNS disease because CaMKII-mediated synaptic dysfunction may develop relatively early in the course of disease progression and thus may be a therapeutic target.

\section{Acknowledgments}

We thank Debbie van Kempen, Bruce Baldwin, Ming Li, Erin Shirk, Chris Bartizal, Brandon Bullock, John Anderson, and John Varrone for expert technical assistance with animal studies. We appreciate helpful discussions with Drs. Janice Clements, Chris Zink, Kelly Gebo, Lucio Gama, Kelly Meulendyke, and Jennifer Schrack.

\section{References}

1. Foley J, Ettenhofer M, Wright M, Hinkin C: Emerging issues in the neuropsychology of HIV infection. Current HIV/AIDS Reports 2008, 5:204-211

2. McArthur JC: HIV dementia: an evolving disease. J Neuroimmunol 2004, 157:3-10

3. Grant I, Sacktor N, McArthur JC: HIV neurocognitive disorders. In The neurology of AIDS (ed 2). Gendelman HE, Grant I, Everall I, Lipton SA, Swindells S (Editors). New York: Oxford University Press, 2005. pp. 359-373

4. Heaton RK, Grant I, Butters N, White DA, Kirson D, Atkinson JH, McCutchan JA, Taylor MJ, Kelly MD, Ellis RJ, Wolfson T, Velin R, Marcotte TD, Hesselink JR, Jernigan TL, Chandler J, Wallace M, Abramson I: The HNRC 500 - Neuropsychology of HIV infection at different disease stages. J Int Neuropsychol Soc 1995, 1:231-251

5. Rippeth JD, Heaton RK, Carey CL, Marcotte TD, Moore DJ, Gonzalez R, Wolfson T, Grant I: Methamphetamine dependence increases risk of neuropsychological impairment in HIV infected persons. J Int Neuropsychol Soc 2004, 10:1-14

6. Masliah E: Mechanisms of synaptic dysfunction in Alzheimer's disease. Histol Histopathol 1995, 10:509-519

7. Masliah E, Heaton RK, Marcotte TD, Ellis RJ, Wiley CA, Mallory M Achim CL, McCutchan JA, Nelson JA, Atkinson JH, Grant I: Dendritic injury is a pathological substrate for human immunodeficiency virusrelated cognitive disorders. HRNC Group. The HIV Neurobehavioral Research Center. Ann Neurol 1997, 42:963-972

8. Arnold SE: Contributions of neuropathology to understanding schizophrenia in late life. Harv Rev Psychiatry 2001, 9:69-76

9. Law AJ, Weickert CS, Hyde TM, Kleinman JE, Harrison PJ: Reduced spinophilin but not microtubule-associated protein 2 expression in the hippocampal formation in schizophrenia and mood disorders: molecular evidence for a pathology of dendritic spines. Am J Psychiatry 2004, 161:1848-1855

10. Zink WE, Anderson E, Boyle J, Hock L, Rodriguez-Sierra J, Xiong H, Gendelman HE, Persidsky Y: Impaired spatial cognition and synaptic potentiation in a murine model of human immunodeficiency virus type 1 encephalitis. J Neurosci 2002, 22:2096-2105

11. Weed MR, Gold LH, Polis I, Koob GF, Fox HS, Taffe MA: Impaired performance on a rhesus monkey neuropsychological testing battery following simian immunodeficiency virus infection. AIDS Res Hum Retroviruses 2004, 20:77-89

12. Miller P, Zhabotinsky AM, Lisman JE, Wang X-J: The Stability of a Stochastic CaMKII Switch: Dependence on the Number of Enzyme Molecules and Protein Turnover. PLoS Biol 2005, 3:e107

13. Lisman J, Schulman H, Cline $\mathrm{H}$ : The molecular basis of CaMKII function in synaptic and behavioral memory. Nat Rev Neurosci 2002, 3:175-190

14. Giese KP, Nikolai FB, Filipkowski RK, Silva AJ: Autophosphorylation at Thr286 of the alpha calcium-calmodulin kinase II in LTP and learning. Science 1998, 279:870-873

15. Glazewski S, Chen CM, Silva A, Fox K: Requirement for alpha-CaMKII in experience-dependent plasticity of the barrel cortex. Science 1996, 272:421-423

16. Glazewski S, Giese KP, Silva A, Fox K: The role of alpha-CaMKII autophosphorylation in neocortical experience-dependent plasticity. Nat Neurosci 2000, 3:911-918

17. Frankland PW, O'Brien C, Ohno M, Kirkwood A, Silva A: AlphaCaMKII-dependent plasticity in the cortex is required for permanent memory. Nature 2001, 411:309-313

18. O'Donnell LA, Agrawal A, Jordan-Sciutto KL, Dichter MA, Lynch DR, Kolson DL: Human immunodeficiency virus (HIV)-induced neurotoxicity: roles for the NMDA receptor subtypes. J Neurosci 2006, 26:981-990

19. Wilcox KS, Buchhalter J, Dichter MA: Properties of inhibitory and excitatory synapses between hippocampal neurons in very low-density cultures. Synapse 1994, 18:128-151 
20. Zink MC, Suryanarayana K, Mankowski JL, Shen A, Piatak M Jr, Spelman JP, Carter DL, Adams RJ, Lifson JD, Clements JE: High viral load in the cerebrospinal fluid and brain correlates with severity of simian immunodeficiency virus encephalitis. J Virol 1999, 73:10480-10488

21. Mankowski JL, Queen SE, Kirstein LM, Spelman JP, Laterra J, Simpson IA, Adams RJ, Clements JE, Zink MC: Alterations in blood-brain barrier glucose transport in SIV-infected macaques. J Neurovirol 1999, 5:695-702

22. Mankowski JL, Queen SE, Tarwater PJ, Adams RJ, Guilarte TR: Elevated peripheral benzodiazepine receptor expression in simian immunodeficiency virus encephalitis. J Neurovirol 2003, 9:94-100

23. Chen W, Sulcove J, Frank I, Jaffer S, Ozdener H, Kolson DL: Development of a human neuronal cell model for human immunodeficiency virus (HIV)-infected macrophage-induced neurotoxicity: apoptosis induced by HIV type 1 primary isolates and evidence for involvement of the bcl-2/bcl-xl-sensitive intrinsic apoptosis pathway. J Virol 2002, 76:9407-9419

24. Arundine M, Tymianski M: Molecular mechanisms of calcium-dependent neurodegeneration in excitotoxicity. Cell Calcium 2003, 34:325-337

25. Haughey NJ, Mattson MP: Calcium dysregulation and neuronal apoptosis by the HIV-1 proteins Tat and gp120. J Acquir Immune Defic Syndr 2002, 31:55-61

26. Ellis R, Langford D, Masliah E: HIV and antiretroviral therapy in the brain: neuronal injury and repair. Nat Rev Neurosci 2007, 8:33-44

27. Wiley CA, Masliah E, Morey M, Lemere C, DeTeresa R, Grafe M, Hansen L, Terry R: Neocortical damage during HIV infection. Ann Neurol 1991, 29:651-657

28. Masliah E, Terry RD, Alford M, DeTeresa R: Quantitative immunohistochemistry of synaptophysin in human neocortex: an alternative method to estimate density of presynaptic terminals in paraffin sections. J Histochem Cytochem 1990, 38:837-844

29. Saito S, Kobayashi S, Ohashi Y, Igarashi M, Komiya Y, Ando S: Decreased synaptic density in aged brains and its prevention by rearing under enriched environment as revealed by synaptophysin contents. J Neurosci Res 1994, 39:57-62

30. Gray BC, Siskova Z, Perry VH, O'Connor V: Selective presynaptic degeneration in the synaptopathy associated with ME7-induced hippocampal pathology. Neurobiol Dis 2009, 35:63-74

31. Masliah E, Mallory M, Alford M, DeTeresa R, Hansen LA, McKeel DW Jr, Morris JC: Altered expression of synaptic proteins occurs early during progression of Alzheimer's disease. Neurology 2001, 56:127-129

32. Tanaka S, Saito M, Morimatsu M, Ohama E: Immunohistochemical studies of the PrP(CJD) deposition in Creutzfeldt-Jakob disease. Neuropathology 2000, 20:124-133

33. Gonzalez RG, Cheng LL, Westmoreland SV, Sakaie KE, Becerra LR, Lee PL, Masliah E, Lackner AA: Early brain injury in the SIV-macaque model of AIDS. AIDS 2000, 14:2841-2849
34. Bissel SJ, Wang G, Ghosh M, Reinhart TA, Capuano S 3rd, Stefano Cole K, Murphey-Corb M, Piatak M Jr, Lifson JD, Wiley CA: Macrophages relate presynaptic and postsynaptic damage in simian immunodeficiency virus encephalitis. Am J Pathol 2002, 160:927-941

35. Wu HY, Hsu FC, Gleichman AJ, Baconguis I, Coulter DA, Lynch DR: FYN-mediated phosphorylation of NR2B TYR-1336 controls calpainmediated NR2B cleavage in neuron and heterologous systems. J Biol Chem 2007, 282:20075-20087

36. Churn SB, Limbrick D, Sombati S, DeLorenzo RJ: Excitotoxic activation of the NMDA receptor results in inhibition of calcium/calmodulin kinase II activity in cultured hippocampal neurons. J Neurosci 1995, 15:3200-3214

37. Zhou Y, Takahashi E, Li W, Halt A, Wiltgen B, Ehninger D, Li GD, Hel JW, Kennedy MB, Silva AJ: Interactions between the NR2B Receptor and CaMKII modulate synaptic plasticity and spatial learning. J Neurosci 2007, 27:13843-13853

38. Silva AJ, Paylor R, Wehner JM, Tonegawa S: Impaired spatial learning in alpha-calcium calmodulin kinase II mutant mice. Science 1992, 257:206-211

39. Silva AJ, Rosahl TW, Chapman PF, Marowitz Z, Friedman E, Frankland PW, Cestari V, Cioffi D, Sudhof TC, Bourtchuladze R: Impaired learning in mice with abnormal short-lived plasticity. Curr Biol 1996, 6:1509-1518

40. Hayashi N, Matsubara M, Jinbo Y, Titani K, Izumi Y, Matsushima N: Nef of HIV-1 interacts directly with calcium-bound calmodulin. Protein Sci 2002, 11:529-537

41. Churn SB, Taft WC, Billingsley MS, Blair RE, DeLorenzo RJ: Temperature modulation of ischemic neuronal death and inhibition of calcium/calmodulin-dependent protein kinase II in gerbils. Stroke 1990, 21:1715-1721

42. Shackelford DA, Yeh RY, Hsu M, Buzsaki G, Zivin JA: Effect of cerebral ischemia on calcium/calmodulin-dependent protein kinase II activity and phosphorylation. J Cereb Blood Flow Metabol 1995, $15: 450-461$

43. Waxham NM, Grotta CJ, Silva JA, Strong R, Aronowski J: Ischemiainduced neuronal damage: a role for calcium/calmodulin-dependent protein kinase II. J Cereb Blood Flow Metabol 1996, 16:1-6

44. Park JK, Namgung U, Lee CJ, Park JO, Jin SH, Kwon OB, Ko SR, Kim SW, Kang EJ, Ko JH, Lee SM, Kim DH, Won MH: Calcium-independent CaMKII activity is involved in ginsenoside Rb1-mediated neuronal recovery after hypoxic damage. Life Sci 2005, 76:1013-1025

45. Novak G, Seeman P, Tallerico T: Increased expression of calcium/ calmodulin-dependent protein kinase $\| \beta$ in frontal cortex in schizophrenia and depression. Synapse 2006, 59:61-68

46. Kaul M, Garden GA, Lipton SA: Pathways to neuronal injury and apoptosis in HIV-associated dementia. Nature 2001, 410:988-994

47. Bellizzi MJ, Lu SM, Gelbard HA: Protecting the synapse: evidence for a rational strategy to treat HIV-1 associated neurologic disease. J Neuroimmune Pharmacol 2006, 1:20-31 